Literatur werden ebenso skizziert wie die örtlichen Buchhandelsstrukturen. Abgerundet werden diese kondensierten Ausführungen durch Einblicke in die Hauptcharakteristika des zeitgenössischen englischen und deutschen Buchhandels. Es folgen elf Kapitel zu ausgewählten deutschsprachigen Mitgliedern des Londoner Buchhandels, deren Fallbeispiele in die zuvor skizzierten Gesamtentwicklungen integriert sind. In unterschiedlichem Umfang werden folgende Personen in ihren Netzwerkstrukturen und Handelsaktivitäten kontextualisiert: Johann Christian Jacobi, Johann Christoph Haberkorn, Johann Andreas Linde, Christlieb Gottreich Seyffert, Carl Heydinger, James Remnant, J.L.W. Gebhardt, Heinrich Escher, Constantin Geisweiler, Johann Benjamin Vogel und Gottlieb Schulze. Zusätzlich zu diesen exemplarischen Zugängen bietet Jefcoate hiernach noch einen breiteren Fokus, der als „Weitere Drucker, Buchhändler, Buchbinder und Kunsthändler“ (Kapitel 17) betitelt ist. Eine Bilanzierung der „Strukturen und Bedeutung des deutschen Anteils am Londoner Buchhandel im 18. Jahrhundert" (Kapitel 18) sowie eine Bibliografie der in deutscher Sprache und von deutschsprachigen Buchhändler in London veröffentlichten Drucke (Kapitel 19) runden den inhaltlichen Teil der Studie ab. Das umfangreiche Quellen- und Literaturverzeichnis (Kapitel 20) sowie ein Personen- und Institutionenregister (Kapitel 21) erhöhen die Nutzerfreundlichkeit der vorgelegten, in ihrer Fülle den Leser fast erschlagenden Forschungen.

Im Vorwort vermutet Jefcoate bescheiden, dass durch seine Forschung „ein völlig neues Bild“ entstehe - da hat er Recht. Die mehr als 600 Seiten, deren empirische Basis in 25 Jahren heranreifen durfte, verändern tatsächlich unsere bisherigen Vorstellungen von den „deutschen“ Konturen des Buchhandelns im London des 18. Jahrhunderts. Als wichtiges Nachschlagewerk wird die Studie wohl für lange Zeit fungieren, auch wenn einige der präsentierten Forschungsdeutungen, etwa zum Kulturtransfer oder zu den Öffentlichkeitsstrukturen, in den letzten Jahren deutlich andere Bewertungen und Veränderungen erlebt haben.
The German book in Wolfenbüttel and abroad: studies presented to Ulrich Kopp in his retirement. Ed. by William A. Kelly and Jürgen Beyer. (Studies in reading and book culture; 1) Tartu: University of Tartu Press, 2014. 344 S., Ill. ISBN 978-9949-32-494-1, \$44,00

Besprochen von Prof. Dr. Siegfried Schmidt: Stellvertretender Leiter, Erzbischöfliche Diözesan- und Dombibliothek Köln mit Bibliothek St. Albertus Magnus, Kardinal-Frings-Str. 1-3, D-50668 Köln, E-Mail: Siegfried.Schmidt@Erzbistum-Koeln.de

\section{DOI 10.1515/bfp-2016-0056}

Ulrich Kopp, dem dieser Sammelband gewidmet ist, verbrachte seine gesamte, vier Jahrzehnte umfassende Berufstätigkeit als ausgebildeter Diplom-Bibliothekar an der Herzog-August-Bibliothek (HAB) in Wolfenbüttel und machte sich dort als Experte für die Erfassung und Erschließung alter Drucke, etwa durch seine Zuarbeit für das Verzeichnis der im deutschen Sprachgebiet erschienenen Drucke des XVI. Jahrhunderts (VD 16), einen Namen. Aus Anlass seines Ausscheidens aus dem aktiven Dienst haben ihm ehemalige Kollegen der HAB und in der buchkundlichen Forschung Tätige, die sicherlich oftmals von seinem bibliografischen Fachwissen während eines Forschungsaufenthaltes in Wolfenbüttel oder im kollegialen Austausch profitiert haben, den vorliegenden Sammelband gewidmet. Er vereint insgesamt 13 buchkundliche Beiträge, die zeitlich vorwiegend in das 16. und 17. Jahrhundert fallen und von denen einige hier näher besprochen werden sollen.

Den Strauß der Aufsätze eröffnet Werner Arnold, vor seiner Pensionierung im Jahre 2009 stellvertretender Direktor in Wolfenbüttel. Arnold beschreibt am Beispiel von Augsburg und Nürnberg, wie nach dem Dreißigjährigen Krieg Buchagenten des Herzogs August des Jüngeren in den süddeutschen Reichsstädten Handschriften und Inkunabeln auf dem Antiquariatsmarkt aufkauften, von denen sich verarmte Stadtbewohner unterschiedlicher Profession trennen mussten. Dazu hat er akribisch einen Katalog der ermittelten Vorbesitzer bzw. Buchagenten, die als Verkäufer auftraten, erstellt und durch ein Register erschlossen. Mit seiner quellengestützten Untersuchung kann Arnold exemplarisch die Aussage untermauern, dass „die negativen Folgen des Krieges [...] zu einer umfangreichen Wanderung von Buchbeständen aus dem Süden in den Norden Deutschlands geführt und die Bibliotheca Augusta quantitativ und qualitativ ganz wesentlich bereichert [haben]" (S. 14).

Zwei weitere Kollegen aus Kopps ehemaliger Wirkungsstätte, Christoph und Gundula Boveland, beschäftigen sich mit der Problematik unfirmierter Drucke des 
17. Jahrhunderts. Zu Recht betonen sie, dass es aus nationalbibliografischer Sicht wünschenswert wäre, wenn man möglichst viele Drucke einer Offizin zuordnen könnte, um so Buchproduktion und Handelsbeziehungen im Buchdruck der Frühen Neuzeit sichtbar zu machen. Anders als beim Druck der Inkunabeln hilft die Typenforschung hier aber kaum weiter, „da die von den Offizinen verwendeten Schriften nicht mehr von ihnen selbst, sondern überwiegend von unabhängigen Schriftgießereien hergestellt wurden“ (S. 83). Im Folgenden zeigen sie in ihrem Aufsatz am Beispiel einer bestimmten Schlussvignette, „dem Bär im Akanthus“, die in Drucken, die um die Mitte des 17. Jahrhunderts entstanden sind, häufiger anzutreffen ist, auf, dass die Einbeziehung des ornamentalen Schmucks der Drucke in dem Bemühen, einen unfirmierten Druck einer Offizin zuzuordnen, ein vielversprechender neuer Forschungsansatz sein kann. Da sich ein bestimmtes Bildmotiv bei der Verwendung durch verschiedene Drucker oftmals nur in Nuancen unterscheidet, sind größere Forschungserfolge hier allerdings erst zu erwarten, wenn es Rechercheinstrumente gibt, die es erlauben, große Mengen digitalisierter Drucke sehr präzise nach bestimmten Bildmustern zu durchsuchen.

Den wohl größten Untersuchungsrahmen steckt der Beitrag von Christoph Reske ab, der schon länger am Institut für Buchwissenschaft in Mainz tätig ist, sich dort 2014 habilitiert hat und der in den letzten Jahren auf äußerst verdienstvolle Weise das traditionsreiche Standardwerk von Josef Benzing „Die Buchdrucker des 16. und 17. Jahrhunderts im deutschen Sprachgebiet" neu bearbeitet und fortgeschrieben hat. Auf diesem Erfahrungshintergrund kann Reske es wagen, eine Übersicht über Druckorte und Druckereien im deutschen Sprachgebiet im 16. Jahrhundert anzubieten. Es wird hier eine hoch interessante Gesamtschau geliefert, etwa wenn der Autor zu dem Ergebnis kommt, dass für das 16. Jahrhundert derzeit 202 Druckorte bekannt sind. Bei einer geschätzten Gesamtzahl von 3500 Städten (1500), darunter 3000 Klein- und Kleinststädte mit weniger als 1000 Einwohnern, bedeutet dies, dass im 16. Jahrhundert „nur etwa sechs Prozent der Städte im deutschen Sprachgebiet Druckereien in ihren Mauern [besaßen]“ (S. 281). Der Buchdruck war also auf diese Gesamtzahl bezogen eher eine Ausnahmeerscheinung im städtischen Gewerbe und konzentrierte sich vordergründig vor allem auf große Städte. Köln mit 102 Druckereien und Nürnberg mit 79 Druckereien (jeweils ca. 40000 Einwohner um 1600) nehmen hier die Spitzenstellung ein. Allerdings war nicht primär die Einwohnerzahl bestimmend für die Frage der Existenz von Druckereien innerhalb der Stadtmauern: „Vielmehr muss ein Zusammenhang mit anderen Faktoren bestehen, dem politi- schen, ökonomischen, intellektuellen und religiösen Status, der sich meist im Stadttypus widerspiegelt“ (S. 283). Es waren vor allem die zu dieser Zeit wirtschaftlich noch prosperierenden Reichsstädte und einige Universitätsstädte, in denen Drucker über längere Zeit wirtschaftlich erfolgreich ihr Gewerbe ausübten, während etwa Residenzstädte, ähnlich wie „die Bergstädte, Exilantenstädte sowie die einer Landeshoheit unterstellten Städte [...] für eine rein ökonomisch motivierte Druckereiansiedlung weitgehend unattraktiv [waren]“ (S. 299).

Einen wertvollen Beitrag $\mathrm{zu}$ dem eigenen Arbeitsgebiet des Gefeierten liefert einer der beiden Herausgeber des Sammelbandes, William A. Kelly, der an der National Library of Scotland tätig war und der als absoluter Fachmann hinsichtlich der retrospektiven Erfassung deutscher und niederländischer Druckschriften in schottischen Bibliotheken gilt. Kelly geht in seinem Beitrag kurz auf die Entstehungsgeschichte des VD 16 ein, das ja, um das angestrebte Ziel der Vollständigkeit zu erreichen, bereits in der Vergangenheit die Zahl der beteiligten Bibliotheken enorm vergrößerte und auch räumlich weit über das ehemalige deutsche Sprachgebiet hinaus ging. Rückblickend stellt er dabei kritisch fest, dass die an der Planung des VD 16 Beteiligten trotz vorliegender Beweise des Gegenteils „were influenced by an unvoiced nationalist spirit which made them imagine that all relevant imprints could be found within Germany" und schließt daran die Frage an „If less than half of the early bibliographical output of Austria could not be found among the holdings of the Austrian National Library, why should one expect anything different from Germany?“ (S. 181). Kelly kann anhand von Beispielen aus den Beständen der National Library of Scotland belegen, dass sich auch in schottischen Bibliotheken weitere unikale Drucke für das VD 16 finden und wertvolle bibliografische Hinweise für dieses retrospektive Katalogwerk geliefert werden können. Auf ähnliche Weise kann Hans-Jörg Künast am Beispiel der im Stadtarchiv Augsburg aufbewahrten Kalendersammlung des Friedrich Endorfer d. Ä. exemplarisch zeigen, dass auch Archive einen interessanten Beitrag zur Komplettierung des VD 16 und VD 17 bieten können: Gerade Almanache, die wie andere Gattungen der Gebrauchsliteratur nur sehr lückenhaft in den Bibliotheken aufbewahrt wurden, finden sich oftmals in größerer Zahl in Archivbeständen.

Bekanntlich waren Reformation und die sich anschließende Konfessionalisierung Katalysatoren für den Buchdruck, da seit dem ersten Drittel des 16. Jahrhunderts in ungeheurer Zahl von den verschiedenen Glaubensrichtungen und Konfessionen Lehr-, Streit- und Verteidigungsschriften auf den Markt geworfen wurden. So wundert es 
nicht, dass einige Aufsätze auch dieser Thematik des Buchdrucks im Zeitalter der Konfessionalisierung zugeordnet werden können. Exemplarisch ist hier Robert Kolbs Beitrag „The printer's funeral sermon: recalling the contributions of the printer in Wittenberg Reformation“ $\mathrm{zu}$ nennen. Etliche weitere Beiträge sind von ihrem Charakter her auf einzelne Drucke, Druckorte oder Themen bezogene Spezialuntersuchungen.

Ungewöhnlich ist der Erscheinungsort dieser gründlich lektorierten und mit einem Personen- und Ortsregister ausgestatteten Festschrift, die eine neue Schriftenreihe der traditionsreichen estnischen Universität Tartu begründet. Der zweite Herausgeber des Bandes, Jürgen Beyer, ist dort wissenschaftlicher Mitarbeiter und dürfte diese Publikationsoption besorgt haben. Mit seiner Untersuchung $\mathrm{zu}$ „Undeutsche(n) Bibeln für Deutsche? Zur Benutzung der ersten lettischen, dörptestnischen und revalestnischen $\mathrm{Bi}$ beldrucke (1685-1715)“ stellt er aber auch einen inhaltlichen Bezug zum Erscheinungsort dieser gehaltvollen buchkundlichen Aufsatzsammlung her.

\section{Holger Böning; Iwan-Michelangelo D’Aprile; Hanno Schmitt; Reinhart Siegert (Hg.): Selbstlesen -} Selbstdenken - Selbstschreiben. Prozesse der Selbstbildung von „Autodidakten“ unter dem Einfluss von Aufklärung und Volksaufklärung vom 17. bis zum 19. Jahrhundert. Mit 600 Kurzbiographien von Autodidakten im deutschen Sprachraum bis 1850 und Verzeichnissen von Bauernbibliotheken. Bremen: edition lumière, 2015. (Philanthropismus und populäre Aufklärung. Studien und Dokumente; Bd. 10). 552 S., s/w. Abb., Fest geb. ISBN 978-3-943245-35-6. € 44,80

Besprochen von Prof. Dr. Peter Vodosek: Seestraße 89, D-70174 Stuttgart, E-Mail:vodosek@hdm-stuttgart.de

DOI 10.1515/bfp-2016-0057

Im November 2014 fand im Museum Reckahn auf dem Gut des Aufklärers Friedrich Eberhard von Rochow (17341805) eine Tagung statt, die sich der Frage „Was charakterisiert Autodidaktinnen und Autodidakten?“ widmete. ${ }^{1}$ Der voluminöse Tagungsband versammelt Beiträge von 30

$1 \mathrm{Zu}$ Rochow siehe Hanno Schmitt und Frank Tosch (Hg.): Vermunft fürs Volk. Friedrich Eberhard von Rochow 1734-1805 im Aufbruch Preußens. Berlin: Henschel, 2001.
Autoren und kann als das zurzeit umfassendste Kompendium zum Thema Autodidaxe gelten. Der Ansatz ist breit gewählt: Als Autodidakten „sollen vor allem solche Menschen betrachtet werden, denen eine formale, auf institutionellem Weg erlangte höhere Bildung verwehrt war, die aber gleichwohl bemerkenswerte Leistungen vollbracht haben“ (S. 9). Dem ist nicht ganz so, denn der eine oder andere erwarb durchaus eine solche Bildung, wenn auch nicht auf dem Gebiet, auf dem er sich auszeichnete. Gleichwohl, ob man die Definition als erschöpfend betrachtet, sie auf „Bildung durch Selbstbildung“ verkürzt oder von „uneducated people“ spricht, die einzelnen Studien dokumentieren eine Vielfalt individueller Fälle und erschüttern, wie die Herausgeber im Vorwort hervorheben, so manche bisherige Gewissheit. Der Bogen spannt sich vom „wohlpräparierten“ Autodidakten Ulrich Bräker, dem „Armen Mann im Tockenburg“ bis zum aufgeklärten adeligen Gutsbesitzer wie dem bereits erwähnten Herrn von Rochow. Selbst ein Genie wir Joseph Haydn ließe sich darunter noch subsumieren, wenn er von sich sagt: „Ich war von der Welt abgesondert, niemand in meiner Nähe konnte mich an mir selber irre machen und quälen, und so mußte ich original werden“. Entsprechend weitgefasst ist auch der Zeitrahmen, der von der „Entdeckung des gemeinen Mannes in der Aufklärung“ im 18. Jahrhundert (mit Vorläufern bereits im 17.) bis zum Bauernastronomen Friedrich Schmid mit Todesjahr 1962 reicht.

Wie bei einem solchen Thema nicht anders möglich, war die Veranstaltung interdisziplinär angelegt und führte Vertreter aus Geschichtswissenschaft, Germanistik, Buchwissenschaft, Presseforschung, Kirchengeschichte, Astronomie, Pädagogik und anderen mehr zusammen. Die Beiträge sind fünf Themenblöcken zugeordnet. Da ihre große Anzahl eine ausführliche Einzelwürdigung ausschließt, will man nicht den ganzen Band nacherzählen, soll exemplarisch vorgegangen werden.

Themenblock 1 bringt fünf „Einführende Beiträge“. Reinhart Siegerts umfänglicher Aufsatz „Handwerker und Bauern der Goethezeit als Leser, Büchersammler und Autoren“ hat zwar zwei früher erschienene Veröffentlichungen zur Grundlage, die er aber zusammengefügt sowie durch neues Material und durch weitere Gesichtspunkte anreichert. Heinrich Bosse untersucht am Beispiel zweier Lebensgeschichten aus dem 18. Jahrhundert, von Johann Jakob Reiske (1716-1774) und Karl Heinrich Lang (17641835), den Transformationsprozess vom informellen selbstgesteuerten Lernen in den Umkreis von Bildungsinstitutionen. In „einigen philologisch-historischen Betrachtungen“ geht Inge Karl der Begriffsgeschichte des Terminus „Autodidakt" vom ersten Auftauchen in Homers Odyssee bis zu Fritz Mauthner in seinem Wörterbuch der Philosophie nach. 